\title{
Erratum: QCD corrections and long-range mechanisms of neutrinoless double beta decay [Phys. Rev. D 94, 096014 (2016)]
}

\author{
C. Arbeláez, M. González, S. G. Kovalenko, and M. Hirsch
}

(Q) (Received 25 April 2018; published 10 May 2018)

DOI: $10.1103 /$ PhysRevD.97.099904

We have found a mistake in the renormalization prescription for the quark fields we use in the paper. As a consequence, an incorrect expression for the anomalous dimensions was derived and is shown in Eq. (16). The correct result with which to replace Eq. (16) is

$$
\gamma_{i j}=\delta_{i j} \gamma_{j}, \quad \text { with } \quad \gamma_{1}=-3 \gamma_{2}=-6 C_{F}, \quad \gamma_{3}=0 .
$$

These changes modify the numerical values in Eq. (19) to

$$
U_{1}\left(\mu_{0}, \Lambda_{0}\right) \simeq 2.0, \quad U_{2}\left(\mu_{0}, \Lambda_{0}\right) \simeq 0.8, \quad U_{3}\left(\mu_{0}, \Lambda_{0}\right)=1
$$

and the limits in the second column "With QCD" in Table I. Here, we show an updated Table I, taking into account the modifications in Eq. (19). As seen, the numerical changes both in Eq. (19) and, as a result, in Table I are very moderate, about 20\%-30\%, and do not alter our conclusion about the insignificance of the QCD corrections to the long-range mechanism of neutrinoless double beta decay.

\begin{tabular}{lcccc}
\hline & & Without QCD & & With QCD \\
& ${ }^{76} \mathrm{Ge}$ & ${ }^{136} \mathrm{Xe}$ & ${ }^{76} \mathrm{Ge}$ & $1.9 \times 10^{-9}$ \\
\hline$C_{1}^{L}$ & $5.3 \times 10^{-9}$ & $3.7 \times 10^{-9}$ & $2.7 \times 10^{-9}$ & $1.9 \times 10^{-9}$ \\
$C_{1}^{R}$ & $5.3 \times 10^{-9}$ & $3.7 \times 10^{-9}$ & $2.7 \times 10^{-9}$ & $2.8 \times 10^{-10}$ \\
$C_{2}^{L}$ & $3.1 \times 10^{-10}$ & $2.2 \times 10^{-10}$ & $3.9 \times 10^{-10}$ & $7.3 \times 10^{-10}$ \\
$C_{2}^{R}$ & $8.2 \times 10^{-10}$ & $5.7 \times 10^{-10}$ & $2.2 \times 10^{-9}$ & $1.5 \times 10^{-9}$ \\
$C_{3}^{L}$ & $2.2 \times 10^{-9}$ & $1.5 \times 10^{-9}$ & $3.4 \times 10^{-7}$ & $2.4 \times 10^{-7}$ \\
$C_{3}^{R}$ & $3.4 \times 10^{-7}$ & $2.4 \times 10^{-7}$ & & \\
\hline
\end{tabular}

